Zur kritischen Theorie der politischen Gesellschaft 
Olaf Asbach • Rieke Schäfer

Veith Selk • Alexander Weiß (Hrsg.)

\section{Zur kritischen Theorie der politischen Gesellschaft}

Festschrift für Michael Th. Greven zum 65. Geburtstag

Unter Mitarbeit von Kerstin Kock

슬 Springer VS 
Herausgeber

Olaf Asbach,

Rieke Schäfer,

Veith Selk,

Alexander Weiß,

Hamburg, Deutschland

Die Deutsche Nationalbibliothek verzeichnet diese Publikation in der Deutschen Nationalbibliografie; detaillierte bibliografische Daten sind im Internet über http://dnb.d-nb.de abrufbar.

\section{Springer VS}

(c) VS Verlag für Sozialwissenschaften | Springer Fachmedien Wiesbaden 2012

Das Werk einschließlich aller seiner Teile ist urheberrechtlich geschützt. Jede Verwertung, die nicht ausdrücklich vom Urheberrechtsgesetz zugelassen ist, bedarf der vorherigen Zustimmung des Verlags. Das gilt insbesondere für Vervielfältigungen, Bearbeitungen, Übersetzungen, Mikroverfilmungen und die Einspeicherung und Verarbeitung in elektronischen Systemen.

Die Wiedergabe von Gebrauchsnamen, Handelsnamen, Warenbezeichnungen usw. in diesem Werk berechtigt auch ohne besondere Kennzeichnung nicht zu der Annahme, dass solche Namen im Sinne der Warenzeichen- und Markenschutz-Gesetzgebung als frei zu betrachten wären und daher von jedermann benutzt werden dürften.

Einbandentwurf: KünkelLopka GmbH, Heidelberg

Gedruckt auf säurefreiem und chlorfrei gebleichtem Papier

Springer VS ist eine Marke von Springer DE. Springer DE ist Teil der Fachverlagsgruppe Springer Science+Business Media.

www.springer-vs.de 


\section{Inhalt}

$\begin{array}{ll}\text { Vorwort } & 7\end{array}$

Olaf Asbach

Michael Th. Greven und die kritische Theorie der politischen Gesellschaft.

Einige einleitende Bemerkungen

\section{Zu einer Theorie der politischen Gesellschaft}

Karsten Fischer

Selbstbeschränkung in der "politischen Gesellschaft":

Eine Bestandsaufnahme im Werk von Michael Th. Greven

Dirk Jörke

Politikwissenschaft als Möglichkeitswissenschaft

Karsten Malowitz

Michael Th. Grevens kritische Wissenschaft der Politik. Eine Annäherung

Katrin Toens

Pfadabhängigkeit und Kontingenz in der politikwissenschaftlichen

Zeitdiagnose: Nur scheinbare Gegensätze?

Harald Bluhm

Konträre Auffassungen über Politik und Entscheidung.

Michael Th. Greven und Panajotis Kondylis - eine Skizze

\section{Zum Zustand von Politik und Demokratie in der Gegenwartsgesellschaft}

Friedbert W. Rüb

Die Verletzlichkeit der Demokratie - eine Spekulation

Rainer Schmalz-Bruns

Die Rückkehr des Politischen - oder:

Die Demokratie(theorie) in der Politisierungsfalle?

Anna Geis

Das „Schlichtungsverfahren" $\mathrm{zu}$ „Stuttgart 21".

Die neueste demokratische Errungenschaft? 
Hans J. Lietzmann

Die Kontingenz der Repräsentation: Bürgerbeteiligung.

Ulrich von Alemann und Philip Daniel

Politische Beteiligung: Wohin?

Kari Palonen

Der Begriff des Parlamentarismus bei Max Weber

Herfried Münkler

Die optimale Hochschullandschaft

Rainer Eisfeld

Kontingenz des Politischen - apolitische Gesellschaft?

Anmerkungen zum langen "Sonderweg“ der USA

\section{Politik und Demokratie im Prozess von Europäisierung und Globalisierung}

Beate Kohler-Koch

Politische Gesellschaft und demokratische Reformierbarkeit der EU

Edgar Grande

Das europäische Imperium und seine Grenzen

Wilfried von Bredow

Ariane Mnouchkine und Human Security.

Transpositionen des Politischen im Théâtre du Soleil

Louis W. Pauly

Democracy and Globalization

\section{Laudatio}

Frank Benseler

Mnemosyne und Parrhesie

\section{Anhang}

Olaf Asbach

Bibliographie der Schriften von Michael Th. Greven von 1973 bis 2011

Zu den Mitwirkenden 


\section{Vorwort}

Es ist immer eine Gratwanderung, aus Anlass eines Jubiläums oder Geburtstages eine Festschrift für einen Wissenschaftler vorzulegen. Denn die Spannung zwischen der persönlicher Würdigung des Geehrten, die eine solche Festschrift einerseits leisten möchte, und der Überzeugung, dass eine solche Würdigung vor allem in Form einer fachwissenschaftlichen Auseinandersetzung mit ihm und den ihn umtreibenden Fragen und Problemen geschehen kann, ist nicht leicht zu überbrücken. Deshalb freuen sich die Herausgeber/in, dass sich all diejenigen, die zu diesem Band beigetragen haben, auf dieses Wagnis eingelassen haben, geleitet von dem Wunsch, die wissenschaftliche Arbeit und damit auch die Person Michael Th. Grevens zu würdigen.

Mit dem 65. Geburtstag von Michael Th. Greven, der den Anlass der vorliegenden Festschrift bildet, ist zugleich der Abschluss seiner Laufbahn als Universitätsprofessor verknüpft. Damit endet eine 34 Jahre währende Zeit, während der er - nach Anfängen als Hochschuldozent an der Gesamthochschule Paderborn - als Hochschullehrer zunächst an der Philipps-Universität Marburg (1978-1991), dann an der Technischen Universität Darmstadt (1991-1995) und schließlich (seit 1995) an der Universität Hamburg gewirkt hat. Unter den sich in diesen Jahrzehnten radikal wandelnden Rahmenbedingungen wissenschaftlicher Forschung und Lehre ist er nicht nur für Generationen von Studierenden und für zahlreiche Nachwuchswissenschaftler/innen eine prägende Persönlichkeit gewesen. Zugleich hat er in zahlreichen Funktionen an der Entwicklung und Vertretung seines Faches, der Politikwissenschaft, mitgewirkt; exemplarisch sei hierfür etwa seine über zwei Jahrzehnte währende Tätigkeit im Vorstand der Deutschen Vereinigung für Politische Wissenschaft genannt, deren Vorsitzender er von 1994 bis 1997 gewesen ist. Angesichts der Vielfalt seiner Aktivitäten als Akademiker wie auch als kritischer Intellektueller, der sich immer auch über die Hochschule und professionelle Aktivitäten hinaus engagiert hat, ist es erstaunlich, dass es Michael Th. Greven gelungen ist, in diesen Jahren ein außerordentlich umfangreiches und vielfältiges Euvre vorzulegen, das in seiner sukzessiv entfalteten Theorie der politischen Gesellschaft ihr Zentrum besitzt und damit eine erkennbare systematische Gestalt gewinnt, die für wissenschaftliche Forschung und Zeitdiagnose wie auch für politisches Handeln gleichermaßen relevant ist.

Es lag deshalb nahe, Michael Th. Greven dadurch zu ehren, dass Kolleginnen und Kollegen, Freundinnen und Freunde sich vorgenommen haben, einerseits einige jener politischen und wissenschaftlichen Themen, Phänomene und Probleme in den Blick zu nehmen, mit denen er sich im Laufe seiner wissenschaftlichen Laufbahn auseinandergesetzt hat. Andererseits und vor allem aber bietet es sich an, die Gelegenheit zu nutzen, um jene theoretischen, analytischen und politischen Fragen, Positionen und The- 
sen, die er dabei vertreten hat, aus je eigener Perspektive zu betrachten und zu untersuchen. Auf diese Weise kann unserer Auffassung nach nicht nur ein Schlaglicht auf Grevens bisheriges Schaffen geworfen werden. Vielmehr können so auch Anregungen dazu gegeben werden, sich auch künftig mit den in seinen Schriften enthaltenen Ansätzen und Erkenntnissen auseinanderzusetzen und sie weiterentwickeln; dass dies nur kritisch geschehen kann, ist für einen kritischen Politik- und Gesellschaftswissenschaftler wie Michael Th. Greven selbstverständlich. Dass diese kritische Auseinandersetzung zudem nicht nur von den zum vorliegenden Band Beitragenden sowie von künftigen Leser/innen und Forschenden geleistet werden wird, ist dabei zu erhoffen: Der letztlich kontingente Umstand, dass er aus beamtenrechtlichen Gründen mit seinem 65 . Geburtstag das offizielle „Dienstende“ als Hochschullehrer erreicht, kann - und sollte unserer Auffassung nach keinesfalls als Abschluss, sondern lediglich als Zwischenstation verstanden werden. Sein Wirken als Wissenschaftler und kritischer Intellektueller wird künftig lediglich in anderen Formen und Bahnen verlaufen. $\mathrm{Zu}$ wünschen bleibt, dass der weitere Weg mehr Zeit und Freiheit lassen wird, um auf der Grundlage des bisher Geleisteten Neues zu schaffen.

Eine Reihe von Kolleginnen und Kollegen, von Freundinnen und Freunden, die ihre Mitarbeit für diese Festschrift zugesagt hatten, konnten ihre Beiträge zu unserem und ihrem größten Bedauern nicht rechtzeitig fertigstellen. Der Erscheinungstermin konnte durch das Vorhaben, Michael Th. Greven die Festschrift am 6. Juli 2012 im Rahmen einer Veranstaltung zum Abschluss seines letzten Semesters als Professor an der Universität Hamburg überreichen zu wollen, nicht lange genug hinausgezögert werden, um diese Beiträge noch aufnehmen zu können.

Die Herausgeber/in danken Kerstin Kock, die bei der Vorbereitung dieses Bandes maßgeblich mitgewirkt hat, unermüdlich und auch unter größtem Zeitdruck unbeirrbar. Ebenso gilt unser Dank Moriz Boje Tiedemann und Nina Eggers für ihre Mitarbeit. Besonders danken möchten wir dem VS-Verlag dafür, diesen Band in sein Programm aufgenommen zu haben. Dabei gilt unserer ganz besonderer Dank Frank Schindler, dem verantwortlichen Lektor für den Bereich Politikwissenschaft, der die Publikation dieses Bandes durch sein Engagement und sein großes Interesse daran überhaupt erst möglich gemacht hat.

Hamburg, im April 2012

Olaf Asbach

Rieke Schäfer

Veith Selk

Alexander Weiß 


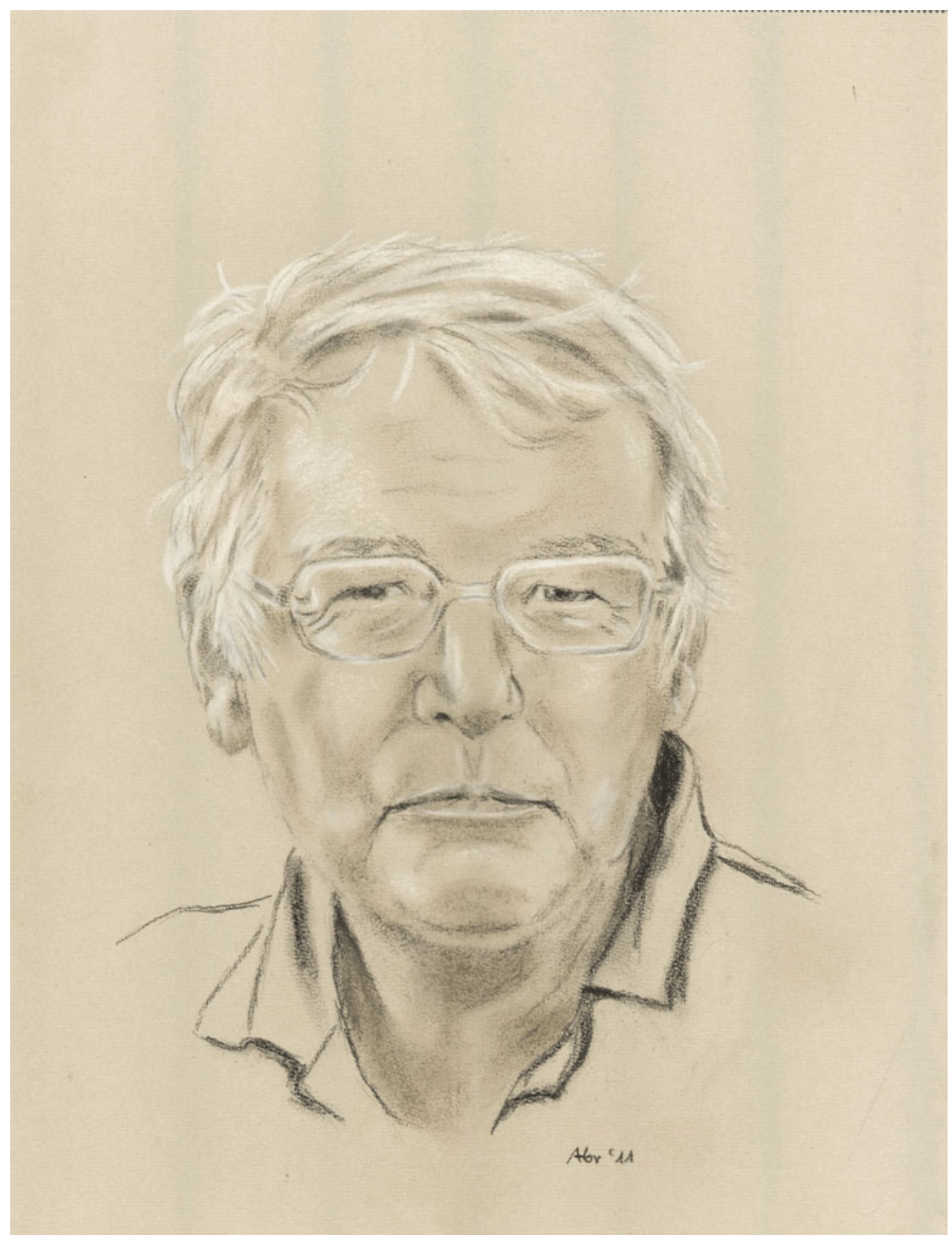

Michael Th. Greven 